\title{
An algebraic approach to problems with polynomial Hamiltonians on Euclidean spaces
}

\author{
D.J. Rowe \\ Department of Physics, University of Toronto \\ Toronto, Ontario M5S 1A\%, Canada
}

\begin{abstract}
Explicit expressions are given for the actions and radial matrix elements of basic radial observables on multi-dimensional spaces in a continuous sequence of orthonormal bases for unitary $\mathrm{SU}(1,1)$ irreps. Explicit expressions are also given for $\mathrm{SO}(N)$-reduced matrix elements of basic orbital observables. These developments make it possible to determine the matrix elements of polynomial and a other Hamiltonians analytically, to within $\mathrm{SO}(N)$ Clebsch-Gordan coefficients, and to select an optimal basis for a particular problem such that the expansion of eigenfunctions is most rapidly convergent.
\end{abstract}

\section{INTRODUCTION}

This paper is concerned with the large class of problems lying between the relatively few that are exactly solvable and others that are only solvable by numerical methods. In particular, algebraic methods are developed for computing the spectral properties of Hamiltonians which are polynomials in the Cartesian position $\left\{x_{i}\right\}$ and momentum $\left\{\hat{p}_{i}=\right.$ $\left.-i \hbar \partial / \partial x_{i}\right\}$ observables of a real Euclidean space $\mathbb{R}^{N}$; polynomials in the inverse square of the radial coordinate are also considered. Restriction to this sub-class of Hamiltonians is because, for them it is appropriate to employ bases of harmonic oscillator or modified oscillator wave functions. Probably a similar treatment can be developed for hydrogenic and modified hyrdrogenic bases which will enable the admission of potentials, like the $1 / r$ potential of the hydrogen atom, which are not rational functions of the Cartesian coordinates.

The most widely studied Hamiltonians of this kind are for exactly solvable central force problems. Some central force problems are solved by use of various realizations of an $\mathrm{SU}(1,1)$ spectrum generating algebra (cf., for example, Refs. [1, 2, 3] for reviews). Others are solved [4] by the so-called factorization method introduced by Schrödinger [5] and employed extensively by Infeld and Hull [6]. (A pedagogical review of the factorization method is given in several texts on quantum mechanics, e.g. 7].) Many special case solutions for power law and inverse power law potentials have also been obtained by the methods of Refs. 8, 9, 10, 11. In an attempt to identify the underlying symmetries of exactly solvable systems, Gendenshtein 12] introduced the criterion of shape invariance and a connection with supersymmetry. Exactly solvable central force problems play an essential role in the current investigation. However, their purpose will be to provide suitable bases in terms of which the matrix elements of a much larger class of Hamiltonians of interest can be determined algebraically.

Underlying the solvability of a central force problem is the factorization of its Hilbert space into a product of radial and orbital subspaces

$$
\mathcal{L}^{2}\left(\mathbb{R}^{N}\right) \simeq \mathcal{L}^{2}\left(\mathbb{R}^{+}, d r\right) \otimes \mathcal{L}^{2}\left(S_{N-1}\right)
$$

where $\mathbb{R}_{+}$is the positive half of the real line,

$$
\mathcal{L}^{2}\left(\mathbb{R}^{+}, d r\right)=\left\{R:\left.r \rightarrow \mathbb{C}\left|\int_{0}^{\infty}\right| R(r)\right|^{2} d r<\infty\right\}
$$

and the elements of $\mathcal{L}^{2}\left(S_{N-1}\right)$ are square integrable functions on the $N-1$ sphere, $S_{N-1} \simeq \mathrm{SO}(N) / \mathrm{SO}(N-1)$, relative to the $\mathrm{SO}(N)$-invariant measure. Thus, because the Hamiltonian for a central force problem is $\mathrm{SO}(N)$-invariant its Schrödinger equation reduces to a one-dimensional radial equation.

More generally the radial $\mathbb{R}^{+}$and orbital $S_{N-1}$ dynamics are coupled by centrifugal forces. Nevertheless, the $\mathrm{SU}(1,1) \times \mathrm{O}(N)$ dynamical group associated with the above factorization continues to provide powerful algebraic (and hence exact) methods for determining the matrix elements of polynomial Hamiltonians in suitable bases. This is of enormous practical value even if it is ultimately necessary to resort to numerical methods for diagonalizing the Hamiltonian matrix. Moreover, it is a huge advantage if bases functions can be found which are close to the desired solutions so that the dimensions of the matrices that have to be diagonalized to achieve a given level of accuracy are relatively small.

With such application in mind, Armstrong [13], Haskell and Wybourne [14], for example, derived expressions for some radial matrix elements in a basis of eigenstates of the three-dimensional harmonic oscillator. It turns out that, for many purposes, other matrix elements are needed and are also needed for higher-dimensional spaces. For example, the nuclear collective model is defined on a five-dimensional space [15, 16, 17]. And, while harmonic oscillator bases 
are appropriate for spherical vibrational systems, they are not the most appropriate for rotational-vibrational systems such as diatomic molecules and non-spherical nuclei.

The current paper gives analytical expressions for the matrix elements of needed observables in a continuous sequence of orthonormal bases for unitary $\mathrm{SU}(1,1) \times \mathrm{O}(N)$ irreps. These irreps include, but are not restricted to, the irreps of the harmonic (discrete) series. Nor are they restricted to a three-dimensional space. Matrix elements of observables that lie in the $\mathrm{SU}(1,1)$ Lie algebra are obtained by algebraic methods. Factorization methods are used to compute the actions and matrix elements of other needed observables. These developments make it possible to select an optimal basis for a particular problem and continue to retain analytical expressions for matrix elements as provided by the harmonic oscillator bases. Basis functions for $\mathcal{L}^{2}\left(S_{N-1}\right)$, which span $\mathrm{O}(N)$ irreps, are commonly referred to as $\mathrm{SO}(N)$-spherical harmonics. They are known for $\mathrm{SO}(3), \mathrm{SO}(4) \simeq \mathrm{SU}(2) \times \mathrm{SU}(2), \mathrm{SO}(5)$ 18, 19], and $\mathrm{SO}(6)$. However, as the following shows, explicit expressions for the wave functions are not needed for the computation of matrix elements. What more is needed, beyond the expressions given in this paper, are the appropriate Clebsch-Gordan coefficients. These are now available in $\mathrm{SO}(3)$-coupled bases for all $N \leq 6$ (cf. section ??).

\section{REPRESENTATIONS OF $\mathrm{SU}(1,1) \times \mathrm{SO}(N)$}

The SU(1,1) Lie algebra (more precisely its complex extension) is spanned by operators $\left\{\hat{S}_{ \pm}, \hat{S}_{0}\right\}$ which satisfy the standard commutation relations

$$
\left[\hat{S}_{-}, \hat{S}_{+}\right]=2 \hat{S}_{0}, \quad\left[\hat{S}_{0}, \hat{S}_{ \pm}\right]= \pm \hat{S}_{ \pm}
$$

A basis for an $\mathrm{SU}(1,1)$ irrep is given by an orthonormal set of states $\{|\lambda \nu\rangle ; \nu=0,1,2, \ldots\}$ which satisfy the identities

$$
\begin{gathered}
\hat{S}_{0}|\lambda \nu\rangle=\frac{1}{2}(\lambda+2 \nu)|\lambda \nu\rangle, \\
\hat{S}_{+}|\lambda \nu\rangle=\sqrt{(\lambda+\nu)(\nu+1)}|\lambda, \nu+1\rangle, \\
\hat{S}_{-}|\lambda \nu\rangle=\sqrt{(\lambda+\nu-1) \nu}|\lambda, \nu-1\rangle .
\end{gathered}
$$

For such an irrep the Casimir invariant

$$
\hat{C}_{\mathrm{SU} 11}=\left(\hat{S}_{0}\right)^{2}-\frac{1}{2}\left(\hat{S}_{+} \hat{S}_{-}+\hat{S}_{-} \hat{S}_{+}\right)=\hat{S}_{0}\left(\hat{S}_{0}-1\right)-\hat{S}_{+} \hat{S}_{-}
$$

takes the value $\lambda(\lambda-2) / 4$, i.e.,

$$
\hat{C}_{\mathrm{SU} 11}|\lambda \nu\rangle=\frac{1}{4} \lambda(\lambda-2)|\lambda \nu\rangle
$$

The $\mathrm{SO}(N)$ Lie algebra is spanned by angular momentum operators $\left\{\hat{\mathcal{L}}_{i j}\right\}$ which are antisymmetric, $\hat{\mathcal{L}}_{i j}=-\hat{\mathcal{L}}_{j i}$, and satisfy the commutation relations

$$
\left[\hat{\mathcal{L}}_{i j}, \hat{\mathcal{L}}_{k l}\right]=-\mathrm{i}\left[\delta_{j k} \hat{\mathcal{L}}_{i l}-\delta_{i l} \hat{\mathcal{L}}_{k j}\right] .
$$

The $\mathrm{SO}(N)$ Casimir operator is defined by

$$
\hat{\Lambda}=\sum_{i<j} \hat{\mathcal{L}}_{i j}^{2}
$$

If an $\mathrm{SO}(N)$ irrep, labelled by $v$, has basis states $\{|v m\rangle\}$, then a basis for an $\mathrm{SU}(1,1) \times \mathrm{SO}(N)$ irrep $(\lambda, v)$ is given by the product states

$$
|\lambda \nu ; v m\rangle \equiv|\lambda \nu\rangle \times|v m\rangle
$$

\section{A. Harmonic series representations}

An explicit realization of the $\mathrm{SU}(1,1)$ Lie algebra is given in terms of harmonic oscillator raising and lowering operators

$$
c_{i}^{\dagger}=\frac{1}{\sqrt{2}}\left(\hat{x}_{i}-\frac{\partial}{\partial x_{i}}\right), \quad c_{i}=\frac{1}{\sqrt{2}}\left(\hat{x}_{i}+\frac{\partial}{\partial x_{i}}\right), \quad i=1, \ldots, N,
$$


by the $\mathrm{O}(N)$-invariant operators

$$
\begin{gathered}
\hat{S}_{+}=\frac{1}{2} c^{\dagger} \cdot c^{\dagger}, \quad \hat{S}_{-}=\frac{1}{2} c \cdot c \\
\hat{S}_{0}=\frac{1}{4}\left(c^{\dagger} \cdot c+c \cdot c^{\dagger}\right)=\frac{1}{2}\left(c^{\dagger} \cdot c+\frac{N}{2}\right)=\frac{1}{2}\left(\hat{n}+\frac{N}{2}\right),
\end{gathered}
$$

where $c \cdot c=\sum_{i} c_{i} c_{i}$ and $\hat{n}=\sum_{i} c_{i}^{\dagger} c_{i}$ is a harmonic oscillator number operator. Similarly the $\mathrm{SO}(N)$ angular momentum operators take the form

$$
\mathcal{L}_{i j}=-\mathrm{i}\left(c_{i}^{\dagger} c_{j}-c_{j}^{\dagger} c_{i}\right)
$$

When the $\mathrm{SU}(1,1)$ and $\mathrm{SO}(N)$ operators are realized in this way, their Casimir invariants are given, respectively, by

$$
4 \hat{C}_{\mathrm{SU} 11}=\hat{n}(\hat{n}+N-2)+\frac{1}{4} N(N-4)-\left(c^{\dagger} \cdot c^{\dagger}\right)(c \cdot c)
$$

and

$$
\hat{\Lambda}=\hat{n}(\hat{n}+N-2)-\left(c^{\dagger} \cdot c^{\dagger}\right)(c \cdot c)
$$

It is seen that when acting on the Hilbert space $\mathcal{L}^{2}\left(\mathbb{R}^{N}\right)$, according to the above-defined realizations, these Casimir invariants are linearly related by the identity

$$
\hat{\Lambda}=4 \hat{C}_{\mathrm{SU} 11}-\frac{1}{4} N(N-4)
$$

in accordance with a well-known duality relationship [20] (also called complementarity [21]). This duality implies that the labels $\lambda$ and $v$ are in one-to-one correspondence. Thus, if the state $|\lambda 0 ; v \sigma\rangle$ satisfies the equations

$$
\hat{S}_{-}|\lambda 0 ; v \sigma\rangle=0, \quad \hat{n}|\lambda 0 ; v \sigma\rangle=v|\lambda 0 ; v \sigma\rangle,
$$

then $\lambda$ takes the value

$$
\lambda=v+N / 2, \quad v=0,1,2, \cdots
$$

Moreover, the eigenvalue of $\hat{\Lambda}$

$$
\hat{\Lambda}|\lambda 0 ; v m\rangle=v(v+N-2)|\lambda 0 ; v m\rangle
$$

signifies that $v$, often referred as seniority, is an $\mathrm{SO}(N)$ angular momentum quantum number.

\section{B. Modified oscillator representations}

The discrete harmonic series of $\mathrm{SU}(1,1)$ irreps which have duality relationships with $\mathrm{SO}(N)$ irreps are invaluable for many purposes. However, there is a continuous series of irreps, which are often more useful. These more general realization of the $\mathrm{SU}(1,1)$ algebra are obtained by expanding the above operators, using eqns. (10),

$$
\hat{S}_{ \pm}=\frac{1}{4}\left[\nabla^{2}+r^{2} \mp\left(2 r \frac{\partial}{\partial r}+N\right)\right], \quad \hat{S}_{0}=\frac{1}{4}\left[-\nabla^{2}+r^{2}\right]
$$

where $r^{2}=\sum_{i} x_{i}^{2}$, and $\nabla^{2}=\sum_{i} \partial^{2} / \partial x_{i}^{2}$ is the Laplacian on $\mathcal{L}^{2}\left(\mathbb{R}^{N}\right)$. The Laplacian has the well-known expansion

$$
\nabla^{2}=\hat{\Delta}-\frac{\hat{\Lambda}}{r^{2}}
$$

where

$$
\hat{\Delta}=\frac{1}{r^{N-1}} \frac{\partial}{\partial r} r^{N-1} \frac{\partial}{\partial r}
$$


In this form the $\mathrm{SU}(1,1)$ operators act as differential operators on the wave functions for the basis states $\{|\lambda \nu ; v m\rangle\}$ of equation (9). These wave functions are conveniently expanded

$$
\Psi_{\lambda \nu v m}(\mathbf{r})=r^{-(N-1) / 2} \mathcal{R}_{\nu}^{\lambda}(r) \mathcal{Y}_{v m}(\omega),
$$

where, for convenience, the factor of $r^{N-1}$ in the $\mathbb{R}^{N}$ volume element $d v(r, \omega)=r^{N-1} d r d \omega$ is absorbed into the definition of the wave functions. Thus, $\left\{\mathcal{R}_{\nu}^{\lambda} ; \nu=0,1,2, \ldots\right\}$ is a basis of radial wave function for $\mathcal{L}^{2}\left(\mathbb{R}^{+}, d r\right)$ and $\left\{\mathcal{Y}_{v m}(\omega)\right\}$ is a basis of spherical harmonics for $\mathcal{L}^{2}\left(S_{N-1}\right)$ defined as eigenfunctions of $\hat{\Lambda}$

$$
\hat{\Lambda} \mathcal{Y}_{v m}=v(v+N-2) \mathcal{Y}_{v m}
$$

With the Laplacian in the form $\nabla^{2}=\hat{\Delta}-\hat{\Lambda} / r^{2}$, the action of the $\mathrm{SU}(1,1)$ operators of equation (11) on the wave functions of eqn. (22) gives

$$
\begin{gathered}
{\left[\hat{S}_{ \pm} \Psi_{\lambda \nu v m}\right](r, \omega)=r^{-(N-1) / 2}\left[\hat{\mathcal{S}}_{ \pm}^{(\lambda)} \mathcal{R}_{\nu}^{\lambda}\right](r) \mathcal{Y}_{v m}(\omega)} \\
{\left[\hat{S}_{0} \Psi_{\lambda \nu v m}\right](r, \theta)=r^{-(N-1) / 2}\left[\hat{\mathcal{S}}_{0}^{(\lambda)} \mathcal{R}_{\nu}^{\lambda}\right](r) \mathcal{Y}_{v m}(\omega)}
\end{gathered}
$$

with $\lambda=v+N / 2$ and

$$
\begin{aligned}
\hat{\mathcal{S}}_{ \pm}^{(\lambda)}= & \frac{1}{4}\left[\frac{d^{2}}{d r^{2}}-\frac{(\lambda-3 / 2)(\lambda-1 / 2)}{r^{2}}+r^{2} \mp\left(2 r \frac{d}{d r}+1\right)\right] \\
& \hat{\mathcal{S}}_{0}^{(\lambda)}=\frac{1}{4}\left[-\frac{d^{2}}{d r^{2}}+\frac{(\lambda-3 / 2)(\lambda-1 / 2)}{r^{2}}+r^{2}\right]
\end{aligned}
$$

Although the $\hat{S}^{(\lambda)}$ operators have been derived for the discrete values of $\lambda=v+N / 2$, they nevertheless satisfy the $\mathrm{SU}(1,1)$ commutation relations

$$
\left[\hat{\mathcal{S}}_{-}^{(\lambda)}, \hat{\mathcal{S}}_{+}^{(\lambda)}\right]=2 \hat{\mathcal{S}}_{0}^{(\lambda)}, \quad\left[\hat{\mathcal{S}}_{0}^{(\lambda)}, \hat{\mathcal{S}}_{ \pm}^{(\lambda)}\right]=\hat{\mathcal{S}}_{ \pm}^{(\lambda)}
$$

for any value of $\lambda$. Moreover, for any real $\lambda>0$, they have a unitary representation and define an orthonormal basis $\left\{\mathcal{R}_{n}^{\lambda}\right\}$ for the Hilbert space $\mathcal{L}^{2}\left(\mathbb{R}_{+}\right)$such that

$$
\begin{gathered}
\hat{\mathcal{S}}_{0}^{(\lambda)} \mathcal{R}_{\nu}^{\lambda}=\frac{1}{2}(\lambda+2 \nu) \mathcal{R}_{\nu}^{\lambda}, \\
\hat{\mathcal{S}}_{+}^{(\lambda)} \mathcal{R}_{\nu}^{\lambda}=\sqrt{(\lambda+\nu)(\nu+1)} \mathcal{R}_{\nu+1}^{\lambda}, \\
\hat{\mathcal{S}}_{-}^{(\lambda)} \mathcal{R}_{\nu}^{\lambda}=\sqrt{(\lambda+\nu-1) \nu} \mathcal{R}_{\nu-1}^{\lambda} .
\end{gathered}
$$

For each $\lambda>0$, the $\hat{S}^{(\lambda)}$ operators span a specific $\mathrm{SU}(1,1)$ representation. This is evidenced by the fact that, for each realization, the $\mathrm{SU}(1,1)$ Casimir operator acquires the unique value $\lambda(\lambda-2) / 4$, i.e.,

$$
\hat{C}_{\mathrm{SU} 11}^{(\lambda)}=\hat{\mathcal{S}}_{0}^{(\lambda)}\left(\hat{\mathcal{S}}_{0}^{(\lambda)}-1\right)-\hat{\mathcal{S}}_{+}^{(\lambda)} \hat{\mathcal{S}}_{-}^{(\lambda)}=\frac{1}{4} \lambda(\lambda-2) \hat{I}
$$

where $\hat{I}$ is the identity.

\section{THE RADIAL WAVE FUNCTIONS}

The $\left\{\mathcal{R}_{\nu}^{\lambda}\right\}$ wave functions for arbitrary $\lambda>0$ are derived as for the standard harmonic oscillator and are given by

$$
\mathcal{R}_{\nu}^{\lambda}(r)=(-1)^{\nu} \sqrt{\frac{2 \nu !}{\Gamma(\lambda+\nu)}} r^{\lambda-1 / 2} \mathrm{~L}_{\nu}^{(\lambda-1)}\left(r^{2}\right) e^{-r^{2} / 2}, \quad \nu=0,1,2, \ldots
$$

The lowest-weight functions $\left\{\mathcal{R}_{0}^{\lambda}\right\}$ are plotted in Fig. 1]

An important characteristic of the radial Hilbert space $\mathcal{L}^{2}\left(\mathbb{R}^{+}, d r\right)$ is that it is independent of the dimensionality of the Euclidean space in which it is embedded. Thus, when $2 \lambda$ is an integer, these functions are equal to the radial wave 


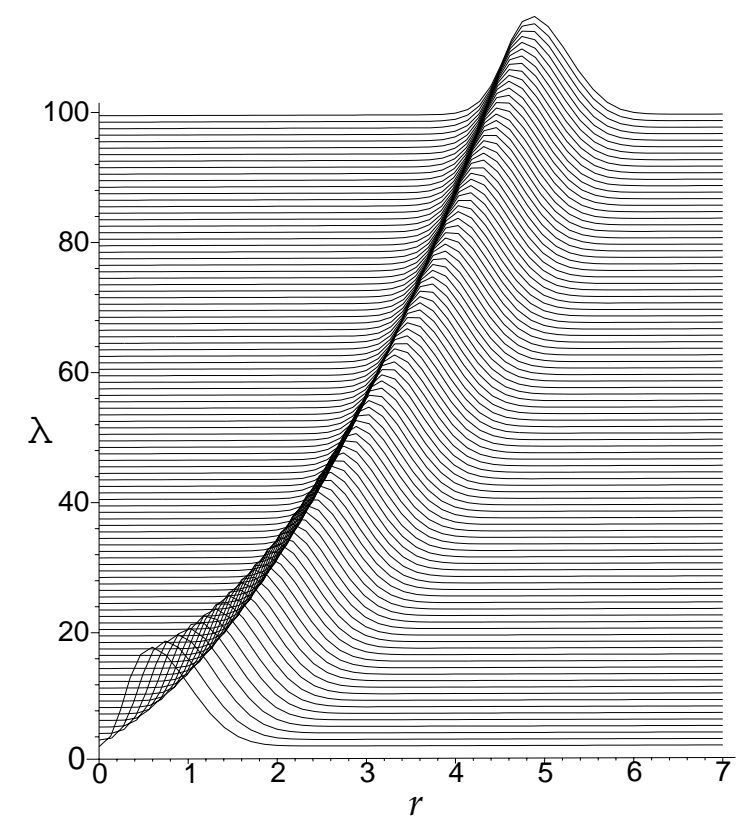

FIG. 1: Radial wave functions $\left\{\mathcal{R}_{0}^{\lambda}\right\}$ plotted as functions of $r$ for a range of values of $\lambda$. (Computed by P.S. Turner [17].)

functions for various $N$-dimensional harmonic oscillators. For example, the wave functions of the simple harmonic oscillator are given by

$$
\begin{array}{rlrl}
u_{2 n}(x) & =\frac{1}{\sqrt{2}} \mathcal{R}_{n}^{1 / 2}(x)=(-1)^{n} \frac{2^{n} n !}{\sqrt{\sqrt{\pi}(2 n) !}} L_{n}^{(-1 / 2)}\left(x^{2}\right) e^{-x^{2} / 2}, & \\
u_{2 n+1}(x) & =\frac{1}{\sqrt{2}} \mathcal{R}_{n}^{3 / 2}(x)=(-1)^{n} \frac{2^{n+1 / 2} n !}{\sqrt{\sqrt{\pi}(2 n+1) !}} x L_{n}^{(1 / 2)}\left(x^{2}\right) e^{-x^{2} / 2}, & & \infty<x<\infty .
\end{array}
$$

Radial wave functions for the spherical $(N=3)$ harmonic oscillator are given by

$$
u_{n l}(r)=\mathcal{R}_{n}^{l+3 / 2}(r)=(-1)^{n} \sqrt{\frac{2 n !}{\Gamma(n+l+3 / 2)}} r^{l+1} \mathrm{~L}_{n}^{(l+1 / 2)}\left(r^{2}\right) e^{-r^{2} / 2} .
$$

In general, the radial wave functions for an $N$-dimensional harmonic oscillator of $\mathrm{SO}(N)$ angular momentum $v$ are given by

$$
u_{n v}(r)=\mathcal{R}_{n}^{v+N / 2}(r)
$$

These identities reflect the fact that harmonic oscillator radial wave functions belong to SU(1,1) irreps which are determined by the angular momentum of the accompanying spherical wave function. For example, eigenstates of the three-dimensional harmonic oscillator of angular momentum $l$ have wave functions which in spherical polar coordinates take the form

$$
\Psi_{n l m}(r, \theta, \varphi)=\frac{1}{r} u_{n l}(r) Y_{l m}(\theta, \varphi)
$$

where $Y_{l m}$ is a spherical harmonic.

When $2 \lambda$ is not an integer, the basis functions $\left\{\mathcal{R}_{n}^{\lambda}\right\}$ are not the radial wave functions of any harmonic oscillator. Nevertheless, they have algebraic properties that are almost as simple as those which are. For many systems, especially models that exhibit rotational as well as vibrational states, it is appropriate to use $\left\{\mathcal{R}_{n}^{\lambda}\right\}$ radial wave functions with $\lambda>v+N / 2$. Such wave functions are eigenfunctions of a modified oscillator Hamiltonian introduced by Davidson 22. for the purpose of describing the rotational-vibrational states of a diatomic molecule. In this model, the atoms of the molecule are 'pushed apart' by enhancing the centrifugal potential by the substitution

$$
\frac{l(l+1)}{r^{2}} \rightarrow \frac{l(l+1)+r_{0}^{4}}{r^{2}}
$$


this is equivalent to replacing the harmonic oscillator potential $r^{2}$ by a potential

$$
V(r)=\frac{r_{0}^{4}}{r^{2}}+r^{2}
$$

which has a minimum at $r=r_{0}$. A five-dimensional version of the Davidson oscillator has been considered as a model for nuclear rotations and vibrations $[23,24,25]$.

\section{RADIAL MATRIX ELEMENTS}

\section{A. Matrix elements obtained from irreps of the $\mathrm{SU}(1,1)$ Lie algebra}

Claim 1: For $\lambda>1$,

$$
\begin{aligned}
& r^{2} \mathcal{R}_{\nu}^{\lambda}(r)= \sqrt{(\lambda+\nu-1) \nu} \mathcal{R}_{\nu-1}^{\lambda}(r)+\sqrt{(\lambda+\nu)(\nu+1)} \mathcal{R}_{\nu+1}^{\lambda}(r) \\
&+(\lambda+2 \nu) \mathcal{R}_{\nu}^{\lambda}(r) \\
& \frac{1}{r^{2}} \mathcal{R}_{\nu}^{\lambda}(r)=\sum_{\mu<\nu} \frac{(-1)^{\mu-\nu}}{\lambda-1} \sqrt{\frac{\nu ! \Gamma(\lambda+\mu)}{\mu ! \Gamma(\lambda+\nu)}} \mathcal{R}_{\mu}^{\lambda}(r) \\
&+\sum_{\mu \geq \nu} \frac{(-1)^{\mu-\nu}}{\lambda-1} \sqrt{\frac{\mu ! \Gamma(\lambda+\nu)}{\nu ! \Gamma(\lambda+\mu)}} \mathcal{R}_{\mu}^{\lambda}(r), \\
& \frac{d^{2}}{d r^{2}} \mathcal{R}_{\nu}^{\lambda}(r)= \sqrt{(\lambda+\nu-1) \nu} \mathcal{R}_{\nu-1}^{\lambda}(r)+\sqrt{(\lambda+\nu)(\nu+1)} \mathcal{R}_{\nu+1}^{\lambda}(r) \\
&-(\lambda+2 \nu) \mathcal{R}_{\nu}^{\lambda}(r)+(\lambda-3 / 2)(\lambda-1 / 2) \frac{1}{r^{2}} \mathcal{R}_{\nu}^{\lambda}(r) . \\
& \nabla^{2} \mathcal{R}_{\nu}^{\lambda}(r) \mathcal{Y}_{v m}(\omega)= \mathcal{Y}_{v m}(\omega)\left[\hat{S}_{+}^{(\lambda)}+\hat{S}_{-}^{(\lambda)}-2 \hat{S}_{0}^{(\lambda)}+\frac{(\lambda-1)^{2}-\left(v+\frac{1}{2} N-1\right)^{2}}{r^{2}}\right] \mathcal{R}_{\nu}^{\lambda}(r) .
\end{aligned}
$$

The first of these equations follows from equation (29) and the observation that $r^{2}$ is an element of the su(1,1) Lie algebra with expansion

$$
r^{2}=\hat{S}_{+}^{(\lambda)}+\hat{S}_{-}^{(\lambda)}+2 \hat{S}_{0}^{(\lambda)} .
$$

Equation (40) is obtained by rexpressing equation (39) as the recursion relation

$$
(\lambda+2 \nu) f_{\mu \nu}^{\lambda}+\sqrt{(\lambda+\nu-1) \nu} f_{\mu, \nu-1}^{\lambda}+\sqrt{(\lambda+\nu)(\nu+1)} f_{\mu, \nu+1}^{\lambda}=\delta_{\mu \nu},
$$

where

$$
f_{\mu \nu}^{\lambda}=\int \mathcal{R}_{\mu}^{\lambda}(r) \frac{1}{r^{2}} \mathcal{R}_{\nu}^{\lambda}(r) \mathrm{d} r=f_{\nu \mu}^{\lambda}
$$

Starting with the value of $f_{00}^{\lambda}=1 /(\lambda-1)$ obtained from equation (72), this equation has solution

$$
f_{\mu \nu}^{\lambda}=\frac{(-1)^{\mu-\nu}}{\lambda-1} \sqrt{\frac{\nu ! \Gamma(\lambda+\mu)}{\mu ! \Gamma(\lambda+\nu)}}, \quad \text { for } \mu \leq \nu .
$$

Equation (41) follows from the identity

$$
\frac{d^{2}}{d r^{2}}-\frac{\left(\lambda-\frac{3}{2}\right)\left(\lambda-\frac{1}{2}\right)}{r^{2}}=\hat{S}_{+}^{(\lambda)}+\hat{S}_{-}^{(\lambda)}-2 \hat{S}_{0}^{(\lambda)} .
$$

The expression for $\nabla^{2}$ is obtained by recalling that, from equations (19), (24) and (25),

$$
\begin{aligned}
& \left\langle\lambda \mu ; v\left|\nabla^{2}\right| \lambda \nu ; v\right\rangle=\left\langle\lambda \mu\left|\left[\hat{S}_{+}^{\left(v+\frac{1}{2} N\right)}+\hat{S}_{-}^{\left(v+\frac{1}{2} N\right)}-2 \hat{S}_{0}^{\left(v+\frac{1}{2} N\right)}\right]\right| \lambda \nu\right\rangle \\
& =\left\langle\lambda \mu\left|\left[\hat{S}_{+}^{(\lambda)}+\hat{S}_{-}^{(\lambda)}-2 \hat{S}_{0}^{(\lambda)}+\frac{\left(\lambda-\frac{1}{2}\right)\left(\lambda-\frac{3}{2}\right)-\left(v+\frac{1}{2} N-\frac{1}{2}\right)\left(v+\frac{1}{2} N-\frac{3}{2}\right)}{r^{2}}\right]\right| \lambda \nu\right\rangle .
\end{aligned}
$$

Matrix elements of higher even powers of $r$ are given by repeated use of equation (39). 


\section{B. Matrix elements obtained by the factorization method}

Similar equations for the operators $r, 1 / r$, and $d / d r$, are obtained by the factorization method [6, []]). Let $A(X)$ and $A^{\dagger}(X)$, where $X$ is a real number, denote the operators

$$
A(X)=\frac{d}{d r}+\frac{X}{r}+r, \quad A^{\dagger}(X)=-\frac{d}{d r}+\frac{X}{r}+r .
$$

Then

$$
\begin{aligned}
& A(X) A^{\dagger}(X)=-\frac{d^{2}}{d r^{2}}+\frac{X(X-1)}{r^{2}}+r^{2}+2 X+1, \\
& A(X)^{\dagger} A(X)=-\frac{d^{2}}{d r^{2}}+\frac{X(X+1)}{r^{2}}+r^{2}+2 X-1
\end{aligned}
$$

It follows that

$$
\begin{gathered}
A\left(\lambda-\frac{1}{2}\right) A^{\dagger}\left(\lambda-\frac{1}{2}\right)=4 \mathcal{S}_{0}^{(\lambda)}+2 \lambda, \\
A^{\dagger}\left(\lambda-\frac{1}{2}\right) A\left(\lambda-\frac{1}{2}\right)=4 \mathcal{S}_{0}^{(\lambda+1)}+2 \lambda-2, \\
A\left(-\lambda+\frac{3}{2}\right) A^{\dagger}\left(-\lambda+\frac{3}{2}\right)=4 \mathcal{S}_{0}^{(\lambda)}-2 \lambda+4, \\
A^{\dagger}\left(-\lambda+\frac{3}{2}\right) A\left(-\lambda+\frac{3}{2}\right)=4 \mathcal{S}_{0}^{(\lambda-1)}-2 \lambda+2 .
\end{gathered}
$$

Equations (52) and (53) give

$$
\begin{aligned}
A\left(\lambda-\frac{1}{2}\right) A^{\dagger}\left(\lambda-\frac{1}{2}\right) \mathcal{R}_{\nu}^{\lambda} & =4(\lambda+\nu) \mathcal{R}_{\nu}^{\lambda}, \\
A^{\dagger}\left(\lambda-\frac{1}{2}\right) A\left(\lambda-\frac{1}{2}\right) \mathcal{R}_{\nu}^{\lambda+1} & =4(\lambda+\nu) \mathcal{R}_{\nu}^{\lambda+1},
\end{aligned}
$$

and hence

$$
\begin{aligned}
A^{\dagger}\left(\lambda-\frac{1}{2}\right) A\left(\lambda-\frac{1}{2}\right)\left[A^{\dagger}\left(\lambda-\frac{1}{2}\right) \mathcal{R}_{\nu}^{\lambda}\right] & =4(\lambda+\nu)\left[A^{\dagger}\left(\lambda-\frac{1}{2}\right) \mathcal{R}_{\nu}^{\lambda}\right] \\
A\left(\lambda-\frac{1}{2}\right) A^{\dagger}\left(\lambda-\frac{1}{2}\right)\left[A\left(\lambda-\frac{1}{2}\right) \mathcal{R}_{\nu}^{\lambda+1}\right] & =4(\lambda+\nu)\left[A\left(\lambda-\frac{1}{2}\right) \mathcal{R}_{\nu}^{\lambda+1}\right] .
\end{aligned}
$$

From these and similar equations obtained from equations (54) and (55), it follows (with a choice of relative phase) that

$$
\begin{gathered}
A^{\dagger}\left(\lambda-\frac{1}{2}\right) \mathcal{R}_{\nu}^{\lambda}=2 \sqrt{\lambda+\nu} \mathcal{R}_{\nu}^{\lambda+1}, \\
\quad A\left(\lambda-\frac{1}{2}\right) \mathcal{R}_{\nu}^{\lambda+1}=2 \sqrt{\lambda+\nu} \mathcal{R}_{\nu}^{\lambda}, \\
A^{\dagger}\left(-\lambda+\frac{3}{2}\right) \mathcal{R}_{\nu}^{\lambda}=2 \sqrt{\nu+1} \mathcal{R}_{\nu+1}^{\lambda-1}, \quad \text { for } \lambda>1 \\
A\left(-\lambda+\frac{3}{2}\right) \mathcal{R}_{\nu+1}^{\lambda-1}=2 \sqrt{\nu+1} \mathcal{R}_{\nu}^{\lambda}, \quad \text { for } \lambda>1 .
\end{gathered}
$$

Thus, it is seen that the $A$ and $A^{\dagger}$ operator act as raising and lowering operators for the radial wave functions in close parallel with the way the standard raising and lowering operators act on harmonic oscillator states; the parallel is exhibited in fig. 2 This observation is exploited in Section \$1 to obtain the actions of the harmonic oscillator raising and lowering operators on general modified oscillator states.

From the identities 60 63), we obtain the results:

Claim 2: For $\lambda>1$

$$
\begin{aligned}
& r \mathcal{R}_{\nu}^{\lambda}(r)=\sqrt{\lambda+\nu-1} \mathcal{R}_{\nu}^{\lambda-1}(r)+\sqrt{\nu+1} \mathcal{R}_{\nu+1}^{\lambda-1}(r), \\
& r \mathcal{R}_{\nu}^{\lambda}(r)=\sqrt{\lambda+\nu} \mathcal{R}_{\nu}^{\lambda+1}(r)+\sqrt{\nu} \mathcal{R}_{\nu-1}^{\lambda+1}(r), \\
& \frac{1}{r} \mathcal{R}_{\nu}^{\lambda}(r)=\sum_{\mu=0}^{\nu}(-1)^{\mu-\nu} \sqrt{\frac{\nu ! \Gamma(\lambda+\mu-1)}{\mu ! \Gamma(\lambda+\nu)}} \mathcal{R}_{\mu}^{\lambda-1}(r), \\
& \frac{1}{r} \mathcal{R}_{\nu}^{\lambda}(r)=\sum_{\mu=\nu}^{\infty}(-1)^{\mu-\nu} \sqrt{\frac{\mu ! \Gamma(\lambda+\nu)}{\nu ! \Gamma(\lambda+\mu+1)}} \mathcal{R}_{\mu}^{\lambda+1}(r),
\end{aligned}
$$




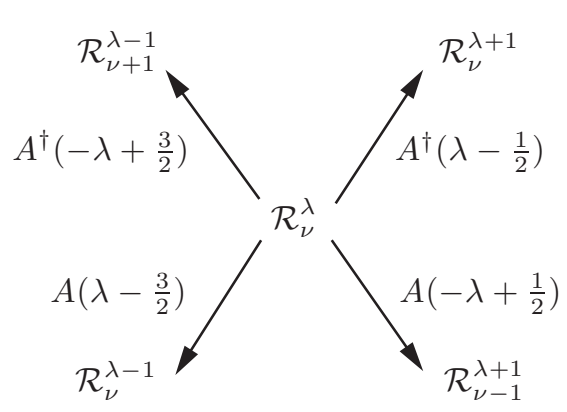

(a)

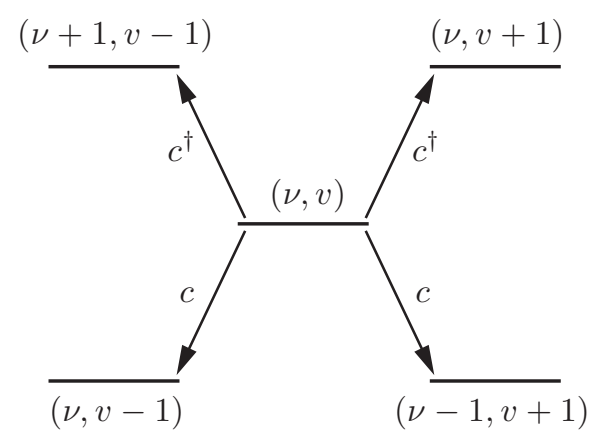

(b)

FIG. 2: The action of the raising and lowering operators of radial wave functions compared to the actions of the standard raising and lowering operators of harmonic oscillator states. Multiplets of harmonic oscillator states of a given energy level are shown as horizontal lines in (b) labelled by radial $\nu$ and $\mathrm{SO}(N)$ angular momentum $v$.

$$
\begin{aligned}
\frac{d}{d r} \mathcal{R}_{\nu}^{\lambda}(r)= & -\sqrt{\nu+1} \mathcal{R}_{\nu+1}^{\lambda-1}(r)+\frac{\nu+\frac{1}{2}}{\sqrt{\lambda+\nu-1}} \mathcal{R}_{\nu}^{\lambda-1}(r) \\
& -\left(\lambda-\frac{3}{2}\right) \sum_{\mu=0}^{\nu-1}(-1)^{\mu-\nu} \sqrt{\frac{\nu ! \Gamma(\lambda+\mu-1)}{\mu ! \Gamma(\lambda+\nu)}} \mathcal{R}_{\mu}^{\lambda-1}(r), \\
\frac{d}{d r} \mathcal{R}_{\nu}^{\lambda}(r)= & \sqrt{\nu} \mathcal{R}_{\nu-1}^{\lambda+1}(r)-\frac{\nu+\frac{1}{2}}{\sqrt{\lambda+\nu}} \mathcal{R}_{\nu}^{\lambda+1}(r) \\
& +\left(\lambda-\frac{1}{2}\right) \sum_{\mu=\nu+1}^{\infty}(-1)^{\mu-\nu} \sqrt{\frac{\mu ! \Gamma(\lambda+\nu)}{\nu ! \Gamma(\lambda+\mu+1)}} \mathcal{R}_{\mu}^{\lambda+1}(r) .
\end{aligned}
$$

The first of these equations follows from equations (61) and (62) and the observation that

$$
2 r=A^{\dagger}\left(-\lambda+\frac{3}{2}\right)+A\left(\lambda-\frac{3}{2}\right) .
$$

The second follows similarly from equations (60) and (63) with

$$
2 r=A^{\dagger}\left(\lambda-\frac{1}{2}\right)+A\left(-\lambda+\frac{1}{2}\right) .
$$

Equation (66) is obtained by expressing equation (65) as a recursion relation

$$
\frac{1}{r} \mathcal{R}_{\nu}^{\lambda}(r)=\frac{1}{\sqrt{\lambda+\nu-1}} \mathcal{R}_{\nu}^{\lambda-1}(r)-\sqrt{\frac{\nu}{\lambda+\nu-1}} \frac{1}{r} \mathcal{R}_{\nu-1}^{\lambda}(r),
$$

which is readily solved to give the desired result. Equation (67) is similarly obtained from equation (64). The last two equations of the claim are obtained from the identity

$$
A(X)-A^{\dagger}(-X)=2\left(\frac{d}{d r}+\frac{X}{r}\right) .
$$

Setting $X=\lambda-3 / 2$, and using equation (66) leads to equation (68). Setting $X=-\lambda+1 / 2$, and using equation (67) leads to equation (69).

\section{An $\mathrm{O}(N)$-parity quantum number}

The above results show that radial matrix elements of type $\left\langle\lambda \mu\left|r^{2}\right| \lambda \nu\right\rangle,\left\langle\lambda \mu\left|1 / r^{2}\right| \lambda \nu\right\rangle$, and $\left\langle\lambda \mu\left|d^{2} / d r^{2}\right| \lambda \nu\right\rangle$ are obtained by use of the $\mathrm{SU}(1,1)$ Lie algebra and those of type $\langle\lambda \pm 1, \mu|r| \lambda \nu\rangle,\langle\lambda \pm 1, \mu|1 / r| \lambda \nu\rangle$, and $\langle\lambda \pm 1, \mu|d / d r| \lambda \nu\rangle$ are obtained by use of the factorization method. However, neither of the methods presented gives matrix elements of the type $\langle\lambda \mu|r| \lambda \nu\rangle,\langle\lambda \mu|1 / r| \lambda \nu\rangle$, and $\langle\lambda \mu|d / d r| \lambda \nu\rangle$. This is because odd powers of $r$ and $d / d r$ do not occur alone in the 
space of polynomial functions of the basic $\left\{x_{i}, p_{i}\right\}$ observables. For example, whereas $r^{2}$ is the quadratic $r^{2}=\sum_{i} x_{i}^{2}$, there is no polynomial expression for $r=\sqrt{\sum_{i} x_{i}^{2}}$. However, terms linear in $r$ and $d / d r$ do occur in combination with orbital functions. For example, when expressed in terms of $\mathbb{R}^{N}$ spherical polar coordinates, the Euclidean coordinates $\left\{x_{i}\right\}$ are of the form $x_{i}=r \mathcal{Q}_{i}(\omega)$, where $\mathcal{Q}_{i}$ is proportional to a $v=1 \mathrm{SO}(N)$ spherical harmonic.

The implications of this observation are conveniently summarized in terms of an $\mathrm{O}(N)$-parity quantum number $\pi=(-1)^{v}$ associated with every $\mathrm{SO}(N)$ irrep of angular momentum $v$. It is seen that even and odd functions of the $\left\{x_{i}\right\}$ coordinates have even and odd $\mathrm{O}(N)$-parity, respectively. It follows that the matrix elements $\left\langle\lambda^{\prime} \mu ; v^{\prime} m\left|\hat{x}_{i}\right| \lambda \nu ; v n\right\rangle$ and $\left\langle\lambda^{\prime} \mu ; v^{\prime} m\left|\hat{p}_{i}\right| \lambda \nu ; v n\right\rangle$ vanish unless $(-1)^{v^{\prime}}=(-1)^{v+1}$. This means that, in the evaluation of polynomial observables, the matrix elements of $r$ and $d / d r$, for example, are only needed between states of opposite parity. Thus, the results of Sections IVA and IVB lead to algebraic expressions for matrix elements of (positive and negative) integer powers of $r$ and $d / d r$ if one chooses basis states $\{|\lambda \nu ; v m\rangle\}$ with $\lambda$ related to $v$ by

$$
\lambda_{v+1}=\lambda_{v} \pm 1 .
$$

The standard relationship $\lambda_{v}=v+N / 2$, given by equation (17) for the harmonic series of SU(1,1) irreps, obviously satisfies this condition. However, there are many other possibilities including, for example, having just two SU $(1,1)$ irreps, one for even- and one for odd $\mathrm{O}(N)$-parity states such that

$$
\lambda_{+}=\lambda_{-} \pm 1
$$

It should be ephasized that Hamiltonians that are not polynomials in $\left\{x_{i}, p_{i}\right\}$ are also of interest. The Hamiltonian of the hydrogen atom, with a singular $1 / r$ potential, is a standard example. However, it would appear that, for such Hamiltonians, the radial wave functions of the modified oscillator do not provide the most appropropriate basis wave functions.

\section{ORBITAL MATRIX ELEMENTS}

This section gives $\mathrm{SO}(N)$-reduced matrix elements of the basic $v=1, \operatorname{SO}(N)$ tensor, $\mathcal{Q}$, defined by the expression

$$
x_{i}=r \mathcal{Q}_{i}
$$

of the Cartesian coordinates in $\mathrm{SO}(N)$ spherical polar coordinates. To make use of these reduced matrix elements one will, in general, need access to $\mathrm{SO}(N)$ Clebsch-Gordan coefficients. The required CG ocefficients are known in $\mathrm{SO}(3)$ $\supset \mathrm{SO}(2)$ coupled bases for $N \leq 6$ (cf. Concluding remarks).

\section{A. $\quad \mathbf{S O}(N)$-reduced matrix elements and a symmetry property}

$\mathrm{SO}(N)$-reduced matrix elements are defined by the Wigner-Eckart theorem

$$
\left\langle v_{3} m_{3}\left|\mathcal{Q}_{m_{2}}\right| v_{1} m_{1}\right\rangle=\left(v_{1} m_{1}, 1 m_{2} \mid v_{3} m_{3}\right)\left\langle v_{3}\|\mathcal{Q}\| v_{1}\right\rangle,
$$

where $\left(v_{1} m_{1}, 1 m_{2} \mid v_{3} m_{3}\right)$ is an $\mathrm{SO}(N)$ Clebsch-Gordan coefficient and $|v m\rangle$ is an orbital basis state whose wave function is an $\mathrm{SO}(N)$ spherical harmonic $\mathcal{Y}_{v m}$. It is then useful to define the $\mathrm{SO}(N)$-coupled action of a tensor operator, e.g. $\mathcal{Q}$, by

$$
\begin{aligned}
{\left[\mathcal{Q} \otimes\left|v_{1}\right\rangle\right]_{v_{3} m_{3}} } & =\sum_{m_{1} m_{2}}\left|v_{3} m_{3}\right\rangle\left\langle v_{3} m_{3}\left|\mathcal{Q}_{m_{2}}\right| v_{1} m_{1}\right\rangle\left(v_{1} m_{1}, 1 m_{2} \mid v_{3} m_{3}\right) \\
& =\left|v_{3} m_{3}\right\rangle\left\langle v_{3}\|\mathcal{Q}\| v_{1}\right\rangle .
\end{aligned}
$$

An application of these definitions leads to the useful expression

$$
\int\left[\mathcal{Y}_{v_{3}}(\omega) \otimes \mathcal{Q} \otimes \mathcal{Y}_{v_{1}}(\omega)\right]_{0} d \omega=k_{v_{3}}\left\langle v_{3}\|\mathcal{Q}\| v_{1}\right\rangle
$$

and, hence, the identity

$$
k_{v_{3}}\left\langle v_{3}\|\mathcal{Q}\| v_{1}\right\rangle=k_{v_{1}}\left\langle v_{1}\|\mathcal{Q}\| v_{3}\right\rangle
$$


where

$$
k_{v}=\int\left[\mathcal{Y}_{v}(\omega) \otimes \mathcal{Y}_{v}(\omega)\right]_{0} d \omega
$$

The value of $k_{v}$ can be inferred, to within a phase factor, by making the expansion

$$
\mathcal{Y}_{v n}=\sum_{n} C_{n m} \mathcal{Y}_{v m}^{*}
$$

which is always possible because the space $\mathcal{L}^{2}\left(S_{N}\right)$ has a real basis. Because spherical harmonics are defined to be an orthonormal basis of orbital wave functions, it follow that

$$
\sum_{n}\left|C_{n m}\right|^{2}=1
$$

and that

$$
k_{v}=\sum_{m^{\prime} m n} C_{n m^{\prime}}(v m, v n \mid 00) \int \mathcal{Y}_{v m^{\prime}}^{*}(\omega) \mathcal{Y}_{v m}(\omega) d \omega=\sum_{m n} C_{n m}(v m, v n \mid 00) .
$$

Now, from the two identities

$$
\sum_{m n}\left|C_{n m}\right|^{2}=d(v), \quad \sum_{m n}(v m, v n \mid 00)^{2}=1,
$$

where $d(v)$ is the dimension of the $\mathrm{SO}(N)$ irrep $v$, we conclude that

$$
C_{n m}=k_{v}(v m, v n \mid 00) \text { and }\left|k_{v}\right|^{2}=d(v) .
$$

It follows that

$$
k_{v}=e^{\mathrm{i} \phi(v)} \sqrt{d(v)}
$$

where $\phi(v)$ is a phase angle, and

$$
\left\langle v_{3}\|\mathcal{Q}\| v_{1}\right\rangle=e^{\mathrm{i}\left(\phi\left(v_{1}\right)-\phi\left(v_{3}\right)\right)} \sqrt{\frac{d\left(v_{1}\right)}{d\left(v_{3}\right)}}\left\langle v_{1}\|\mathcal{Q}\| v_{3}\right\rangle .
$$

It is important to note, that reduced matrix elements are sometimes defined by expressing the Wigner-Eckart theorem in the form

$$
\left\langle v_{3} m_{3}\left|\mathcal{Q}_{m_{2}}\right| v_{1} m_{1}\right\rangle=\left(v_{1} m_{1}, 1 m_{2} \mid v_{3} m_{3}\right) \frac{\overline{\left\langle v_{3}\|\mathcal{Q}\| v_{1}\right\rangle}}{\sqrt{d\left(v_{3}\right)}} .
$$

This adjusted definition of the reduced matrix elements results in a simplification of the symmetry relation (88) to

$$
\overline{\left\langle v_{3}\|\mathcal{Q}\| v_{1}\right\rangle}=e^{\mathrm{i}\left(\phi\left(v_{1}\right)-\phi\left(v_{3}\right)\right)} \overline{\left\langle v_{1}\|\mathcal{Q}\| v_{3}\right\rangle} .
$$

\section{B. Basic reduced matrix elements}

Reduced matrix elements of the basic $v=1, \mathrm{SO}(N)$ tensor, $\mathcal{Q}$, are determined by the expansion

$$
x_{i}=\frac{1}{\sqrt{2}}\left(c_{i}^{\dagger}+c_{i}\right)
$$

and the matrix elements of the harmonic oscillator raising and lowering operators. This expression shows that $\mathcal{Q}$ has non-zero reduced matrix elements $\left\langle v^{\prime}\|\mathcal{Q}\| v\right\rangle$ only for $v^{\prime}=v \pm 1$. 
Let

$$
a^{\dagger}=\frac{1}{\sqrt{2}}\left(c_{1}^{\dagger}+\mathrm{i} c_{2}^{\dagger}\right)
$$

denote the highest weight component of the harmonic oscillator raising operators in which the operators $\left\{c_{i}^{\dagger}\right\}$ of equation (10) are regarded as Cartesian components of a $v=1, \mathrm{SO}(N)$ tensor. Then, with $\lambda_{v}=v+N / 2$, the harmonic oscillator ground state is the state $|0\rangle=\left|\lambda_{0} \nu=0 ; v=m=0\right\rangle$ and a subset of excited states is given by

$$
\left|\lambda_{v} 0 ; v v\right\rangle=\frac{1}{\sqrt{v !}}\left(a^{\dagger}\right)^{v}|0\rangle, \quad v=0,1,2, \cdots
$$

The matrix element

$$
\left\langle\lambda_{v+1} 0 ; v+1, v+1\left|a^{\dagger}\right| \lambda_{v} 0 ; v v\right\rangle=\sqrt{v+1}
$$

then implies that

$$
\left\langle\lambda_{v+1} 0 ; v+1\|r \mathcal{Q}\| \lambda_{v} 0 ; v\right\rangle=\frac{1}{\sqrt{2}}\left\langle\lambda_{v+1} 0 ; v+1\left\|c^{\dagger}\right\| \lambda_{v} 0 ; v\right\rangle=\sqrt{\frac{v+1}{2}}
$$

Thus, we obtain the following result:

Claim 3: $\mathrm{SO}(N)$-reduced matrix elements of the basic $v=1, \mathrm{SO}(N)$ tensor, $\mathcal{Q}$, defined by the expression $x_{i}=r \mathcal{Q}_{i}$ are given by

$$
\left\langle v^{\prime}\|\mathcal{Q}\| v\right\rangle=\sqrt{\frac{v+1}{2 v+N}} \delta_{v^{\prime}, v+1}+e^{\mathrm{i}(\phi(v)-\phi(v-1))} \sqrt{\frac{d(v) v}{d(v-1)(2 v+N-2)}} \delta_{v^{\prime}, v-1} .
$$

The first term of this expression is obtained by factoring out the radial matrix element $\left\langle\lambda_{v}+1,0|r| \lambda_{v} 0\right\rangle=\sqrt{\lambda_{v}}=$ $\sqrt{v+N / 2}$ in equation (94). The second term is obtained from the first by use of the symmetry relationship (88).

For $N=3$, for example, the customary expression of the Cartesian coordinates in terms of $(r, \theta, \varphi)$ spherical polar coordinates

$$
\begin{gathered}
x_{1}=r \sin \theta \cos \varphi, \\
x_{2}=r \sin \theta \sin \varphi, \\
x_{3}=r \cos \theta,
\end{gathered}
$$

defines the Cartesian components $\left\{\mathcal{Q}_{i}\right\}$ of the $N=3$ tensor $\mathcal{Q}$. The dimension of an $\operatorname{SO}(3)$ irrep of angular momentum $l$ (a standard symbol for $\mathrm{SO}(3)$ angular momentum) is given by $d(l)=2 l+1$ and, with the standard phase convention for $\mathrm{SO}(3)$ spherical harmonics, we obtain $e^{\mathrm{i} \phi(l)}=(-1)^{l}$. Thus, claim 3 gives

$$
\left\langle l^{\prime}\|\mathcal{Q}\| l\right\rangle=\sqrt{\frac{l+1}{2 l+3}} \delta_{l^{\prime}, l+1}+\sqrt{\frac{l}{2 l-1}} \delta_{l^{\prime}, l-1} .
$$

For $N=5$, the Weyl dimension formula gives

$$
d(v)=\frac{1}{6}(v+1)(v+2)(2 v+3)
$$

and, with the phase convention $e^{\mathrm{i} \phi(v)}=1$ (used in Ref. [19]), claim 3 gives

$$
\left\langle v^{\prime}\|\mathcal{Q}\| v\right\rangle=\sqrt{\frac{v+1}{2 v+5}} \delta_{v^{\prime}, v+1}+\sqrt{\frac{v+2}{2 v+1}} \delta_{v^{\prime}, v-1} .
$$




\section{COMBINED MATRIX ELEMENTS}

Reduced matrix elements of $x=r \mathcal{Q}$ are obtained by combining the expression for $r$, given in terms of radial raising and lowering operators by equations (70) and (71), with those for the matrix elements of $\mathcal{Q}$, given by claim 2 . In particular, in the harmonic oscillator basis, for which $\lambda_{v}=v+N / 2$ (cf. fig. 22), one obtains:

$$
\begin{gathered}
\left\langle\lambda_{v+1} \nu ; v+1\|x\| \lambda_{v} \nu ; v\right\rangle=\frac{1}{2}\left\langle\lambda_{v+1} \nu\left|A^{\dagger}\left(\lambda_{v}-\frac{1}{2}\right)\right| \lambda_{v}, \nu\right\rangle\langle v+1\|\mathcal{Q}\| v\rangle, \\
\left\langle\lambda_{v+1}, \nu-1 ; v+1\|x\| \lambda_{v} \nu ; v\right\rangle=\frac{1}{2}\left\langle\lambda_{v+1} \nu\left|A\left(-\lambda_{v}+\frac{1}{2}\right)\right| \lambda_{v} \nu\right\rangle\langle v+1\|\mathcal{Q}\| v\rangle, \\
\left\langle\lambda_{v-1}, \nu+1 ; v-1\|x\| \lambda_{v} \nu ; v\right\rangle=\frac{1}{2}\left\langle\lambda_{v-1}, \nu+1\left|A^{\dagger}\left(-\lambda_{v}+\frac{3}{2}\right)\right| \lambda_{v}, \nu\right\rangle\langle v-1\|\mathcal{Q}\| v\rangle, \\
\left\langle\lambda_{v-1} \nu ; v-1\|x\| \lambda_{v} \nu ; v\right\rangle=\frac{1}{2}\left\langle\lambda_{v-1} \nu\left|A\left(\lambda_{v}-\frac{3}{2}\right)\right| \lambda_{v}, \nu\right\rangle\langle v-1\|\mathcal{Q}\| v\rangle .
\end{gathered}
$$

These identities have an immediate generalization.

Claim 4: If $\alpha$ and $\beta$ index any radial wave functions, the $\mathrm{SO}(N)$ reduced matrix elements of the harmonic oscillator raising and lowering operators between states of $\mathrm{SO}(N)$ angular momentum $v$ and $v^{\prime}$ are given by

$$
\begin{aligned}
\left\langle\alpha v^{\prime}\left\|c^{\dagger}\right\| \beta v\right\rangle= & \frac{1}{\sqrt{2}}\left[\left\langle\alpha\left|A^{\dagger}\left(v+\frac{1}{2} N-\frac{1}{2}\right)\right| \beta\right\rangle \delta_{v^{\prime}, v+1}+\left\langle\alpha\left|A^{\dagger}\left(-v-\frac{1}{2} N+\frac{3}{2}\right)\right| \beta\right\rangle \delta_{v^{\prime}, v-1}\right] \\
& \times\left\langle v^{\prime}\|\mathcal{Q}\| v\right\rangle, \\
\left\langle\alpha v^{\prime}\|c\| \beta v\right\rangle= & \frac{1}{\sqrt{2}}\left[\left\langle\alpha\left|A\left(-v-\frac{1}{2} N+\frac{1}{2}\right)\right| \beta\right\rangle \delta_{v^{\prime}, v+1}+\left\langle\alpha\left|A\left(v+\frac{1}{2} N-\frac{3}{2}\right)\right| \beta\right\rangle \delta_{v^{\prime}, v-1}\right] \\
& \times\left\langle v^{\prime}\|\mathcal{Q}\| v\right\rangle .
\end{aligned}
$$

With the substitution $x_{i}=\frac{1}{\sqrt{2}}\left(c_{i}^{\dagger}+c_{i}\right)$, these results follow immediately from equations 100 103) when the radial wave functions are those of the harmonic oscillator basis. However, because the radial wave functions $\left\{\mathcal{R}_{\nu}^{\lambda} ; \nu=\right.$ $0,1,2, \ldots\}$ span the space of radial wave functions for any value of $\lambda$, and because equations (104) and (105) with $|\beta v\rangle=\left|\lambda_{v} \nu ; v\right\rangle$ and $\left|\alpha v^{\prime}\right\rangle=\left|\lambda_{v^{\prime}} \nu^{\prime} ; v^{\prime}\right\rangle$ hold for all values of $\nu$ and $\nu^{\prime}$ they also hold for any radial wave functions.

Because of the way the results of claim 4 are derived, they clearly incorporate the identity

$$
\left\langle\alpha v^{\prime}\|x\| \beta v\right\rangle=\langle\alpha|r| \beta\rangle\left\langle v^{\prime}\|\mathcal{Q}\| v\right\rangle .
$$

What is more significant is that they also give the reduced matrix elements

$$
\begin{aligned}
& \left\langle\alpha, v+1\||\hat{p} \|| \beta v\rangle=-\mathrm{i} \hbar\left\langle\alpha\left|\left[\frac{d}{d r}-\frac{v+\frac{1}{2} N-\frac{1}{2}}{r}\right]\right| \beta\right\rangle\langle v+1\|\mathcal{Q}\| v\rangle,\right. \\
& \langle\alpha, v-1\|\hat{p}\||| \beta v\rangle=-\mathrm{i} \hbar\left\langle\alpha\left|\left[\frac{d}{d r}+\frac{v+\frac{1}{2} N-\frac{3}{2}}{r}\right]\right| \lambda \nu\right\rangle\langle v-1\|\mathcal{Q}\| v\rangle,
\end{aligned}
$$

for the momentum operators

$$
\hat{p}_{i}=-\mathrm{i} \hbar \frac{\partial}{\partial x_{i}}=-\frac{\mathrm{i} \hbar}{\sqrt{2}}\left(c_{i}-c_{i}^{\dagger}\right)
$$

\section{APPLICATION TO CENTRAL FORCE PROBLEMS}

The characteristic property of a central force problem is that its Hamiltonian is $\mathrm{SO}(N)$-invariant. For example, for a diatomic molecule the relevant Hilbert space is the space of $\mathcal{L}^{2}\left(\mathbb{R}^{3}\right)$ wave functions in the relative coordinates of two atoms. For a free molecule in an isotropic space, the Hamiltonian is invariant under $\mathrm{SO}(3)$ rotations. Thus, its Schrödinger equation reduces to an equation in a single radial variable. Moreover, as already noted, the Hilbert spaces of radial wave functions are independent of $N$. Thus, methods developed for the solution of central force problems in $\mathbb{R}^{3}$ apply more generally.

Consider, for example, the Hamiltonian of the quartic oscillator

$$
\hat{H}=-\frac{1}{2} \nabla^{2}+r^{4}
$$


Its spectrum was derived by Bell et al. [27] in two- and three-dimensional spaces by diagonalization in a basis of eigenstates of the harmonic oscillator Hamiltonian

$$
\hat{H}_{\mathrm{HO}}=-\frac{1}{2 a^{2}} \nabla^{2}+\frac{1}{2} a^{2} r^{2}
$$

with $a=1$. It has been considered more recently in five-dimensional space [28]. The addition of quartic terms, which lie in the $\mathrm{SU}(1,1)$ enveloping algebra, to a nuclear collective model Hamiltonian has also been considered by several authors, e.g., 29]. Some low-lying energy levels of the quartic oscillator in three dimensions are shown in Fig. 3 In repeating the calculations of ref. 27] for all $L \leq 6$ energy levels below 250 (in oscillator units), it was found that $21 \mathrm{~s}$ of computer time were needed for each $L$ to compute these energy levels to an accuracy of 1 part in $10^{12}$ when $a=1$ but only $2.6 \mathrm{~s}$ were needed with $a=1.6$.

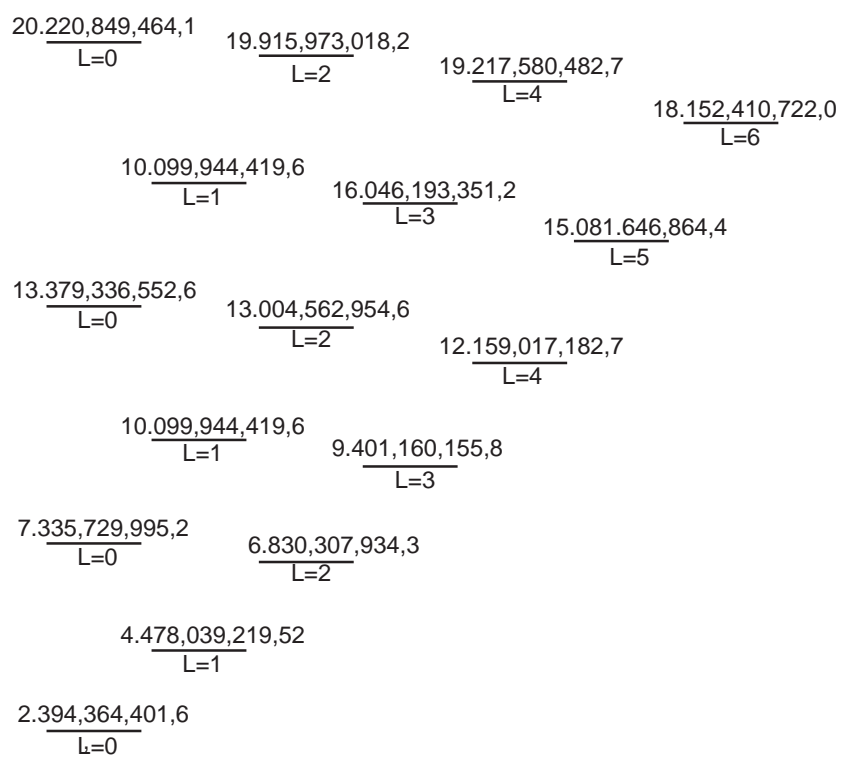

FIG. 3: Low energy levels of the quartic oscillator in three dimensions.

A much larger gain is realized for the low-energy states of potentials with a minimum at a non-zero value of $r$ as the following application to the study of a phase transition in the nuclear collective model shows. Such a calculation was performed originally [26] in a five-dimensional harmonic oscillator basis. However, subsequent studies [17] showed that it can be carried out much more efficiently using modified oscillator bases and the algebraic matrix elements associated with them. This gain in efficiency is particularly relevant, as discussed below, because it makes it practically possible to execute more sophisticated calculations in which the radial and orbital degrees of freedom are coupled.

The Hilbert space for the nuclear collective model is $\mathcal{L}^{2}\left(\mathbb{R}^{5}\right)$ and the Hamiltonian used in Ref. [26] was

$$
\hat{H}(\alpha)=-\frac{1}{2 M} \nabla^{2}+\frac{1}{2} M\left[(1-2 \alpha) r^{2}+\alpha r^{4}\right],
$$

where $r$ is a radial coordinate for $\mathbb{R}^{5}$ and $M$ a mass parameter. This Hamiltonian is interesting because, as $\alpha$ passes through the critical value of 0.5 , a phase transition occurs from a spherical vibrational phase, corresponding to a minimum value of $V(r)$ at $r=0$, to a rotational-vibrational phase for $\alpha>0.5$, corresponding to a minimum value of $V(r)$ at $\sqrt{(2 \alpha-1) /(2 \alpha)}$ (in oscillator units proportional to $\sqrt{M}$ ).

\section{A. Variational calculations}

The benefits from the new develoments are maximized by selecting basis wave functions that are as close as possible to the eigenfunctions. For each value of $\alpha$ in the Hamiltonian, an optimal orthonormal basis of states $|n v m\rangle$ with wave functions of the form

$$
\Phi_{n v m}(r, \theta)=\frac{\sqrt{a_{v}}}{r^{2}} \mathcal{R}_{n}^{\lambda_{v}}\left(a_{v} r\right) \mathcal{Y}_{v m}(\theta)
$$


is obtained by varying the parameters $a_{v}$ and $\lambda_{v}$ for each value of the $\mathrm{SO}(N)$ angular momentum $v$ to minimize the energy expectation values of $\langle 0 v m|\hat{H}(\alpha)| 0 v m\rangle$. The energy-levels obtained from the expectation values

$$
E_{n v m}(\alpha) \approx\langle n v m|\hat{H}(\alpha)| n v m\rangle,
$$

with the $v$-dependent variationally-determined values of $a_{v}$ and $\lambda_{v}$, are shown as functions of $\alpha$ in comparison with

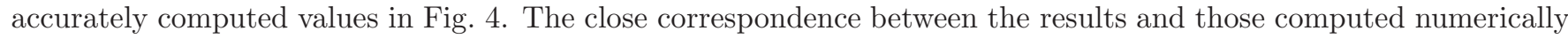
by diagonalization is remarkable. Even in the transition region, where restriction to single basis states is least successful, it does surprisingly well.

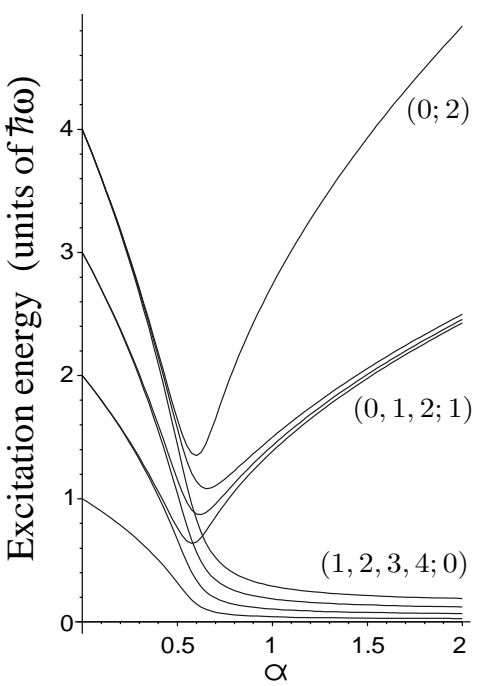

(a)

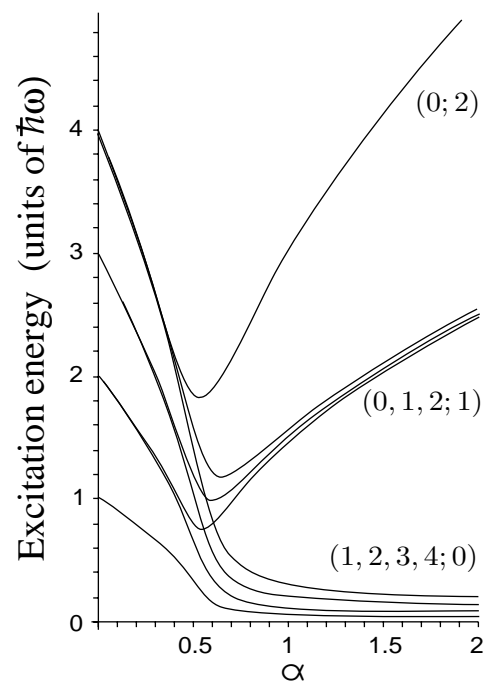

(b)

FIG. 4: Comparison of the low-lying energy levels for the Hamiltonian $\hat{H}(\alpha)$ with $M=100$ as computed (a) by diagonalization and (b) by taking expectation values $\langle n v m|\hat{H}(\alpha)| n v m\rangle$ in single basis states with variationally chosen parameters as described in the text. The numbers shown on the right hand side of each figure are the values of the quantum numbers $\left(v_{1}, v_{2}, \cdots ; \nu\right)$ for each of the levels plotted. More detailed information is given for $\alpha=1.5$ in figure 7 (Figure (a) was computed by P.S. Turner [26].)

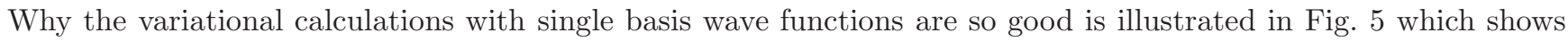
that a potential of the form

$$
V(r)=-b r^{2}+c r^{4}
$$

which has a minimum at $r=r_{0}$, is well fitted in the neighbourhood of its minimum by a potential

$$
W(r)=W_{0}+\frac{r_{0}^{4}}{(a r)^{2}}+(a r)^{2},
$$

with a scale parameter $a$ chosen to give $W(r)$ the curvature of the potential $V(r)$ at $r_{0}$. The radial eigenfunction for the ground state of a Hamiltonian with potential $W(r)$ is given by $\mathcal{R}_{0}^{50}(r)$ (in suitable units of $r$ ) and found to be an excellent approximation to that for the potential $V(r)$.

The values of $a$ and $\lambda$ which minimize the variational ground-state energy are shown in Fig. [6] The figure shows that the wave function is essentially that of a spherical vibrator for $\alpha<0.5$ but that its width increases with $\alpha$ until the point at which the curvature of the potential $V(r)=\frac{1}{2} M\left[(1-2 \alpha) r^{2}+\alpha r^{4}\right]$ at its $r=0$ minimum vanishes. With further increase in $\alpha$, the wave function becomes that of a rotor-vibrator with equilibrium deformation given by $r_{0}=\left[(\lambda-1)^{2}-9 / 4\right]^{1 / 4} / a$

\section{B. Results from numerical diagonalization}

With the analytical expressions given in Sect. IVA variational calculations of the type shown above are easy to implement and give insightful information about the states of the system. Moreover, they are readily extended to obtain more precise results by diagonalization of the Hamiltonian in an optimal basis. 


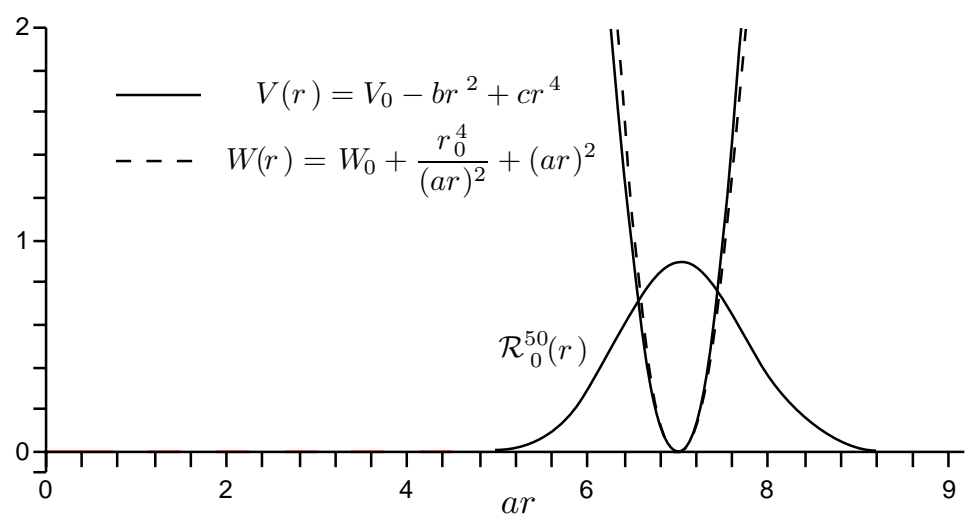

FIG. 5: Comparison of the potentials $V(r)$ and $W(r)$, as defined in the text, for fitted values of $\lambda$ and $a$. $\left\{\mathcal{R}_{0}^{50}\right\}$ is the radial wave function for the ground state of the Hamiltonian with potential $W(r)$.

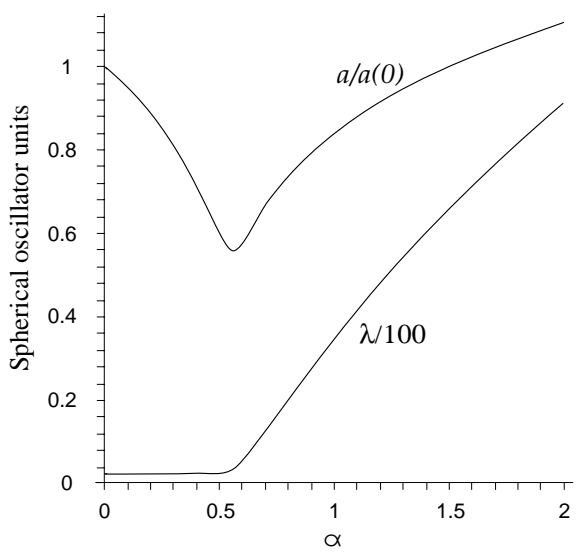

FIG. 6: Values of $\lambda$ and the inverse width parameter $a / a(0)$, where $a(0)=\sqrt{M}$, for the variational ground-state wave functions used in Fig. प (b).

If the objective is to obtain the energy eigenstates for the $n$ lowest-energy states as accurately as possible when computed in a space spanned by the first $N$ states of an ordered basis, then a simple prescription for selecting a near to optimal basis among a set of possible choices is to select the basis which minimizes the computed energy for the highest among the $n$ lowest energy states.

Other prescriptions can obviously be defined such as, for example, minimizing the sum of the energies of the lowest $n$ states. However, it is not clear that much can be gained over the above simple prescription.

For $\alpha \gtrsim 1.5$, for which the model nucleus described by the Hamiltonian $\hat{H}(\alpha)$ of equation (112) is clearly nonspherical for $M=100$, it was found that the energy of the lowest energy state of each $\mathrm{SO}(5)$ angular momentum $v$ was obtained to within $1 \%$ accuracy with just one modified oscillator basis wave function. To achieve this level of accuracy with a conventional harmonic oscillator basis requires $\gtrsim 22$ harmonic oscillator basis wave functions (depending on the value of $\alpha$ ). Fig. 7 shows the excitation energies of the three lowest-energy states for each $v \leq 6$ computed precisely and with just 5 basis states.

The basis states were chosen for the $v=0$ states according to the above given prescription. This prescription could have been followed for each value of $v$. However, in practice, one will want to be able to compute electromagnetic transition matrix elements. Having fixed $\lambda$ for the $v=0$ states (which turned out to be 57) we then chose $\lambda=57$ for all even $v$ and $\lambda=58$ for all odd $v$ for the reasons discussed in Sect. IVC

To obtain parallel results to the same level of accuracy in a harmonic oscillator basis requires $\sim 25$ basis states and a computation that takes $\sim 10$ times as long. For practical purposes, a reduction in the required number of radial basis states is particularly advantageous in treating systems in which there is a coupling between the orbital and radial degrees of freedom; this is because the product of the needed number of radial and orbital basis states can reach a large number. 

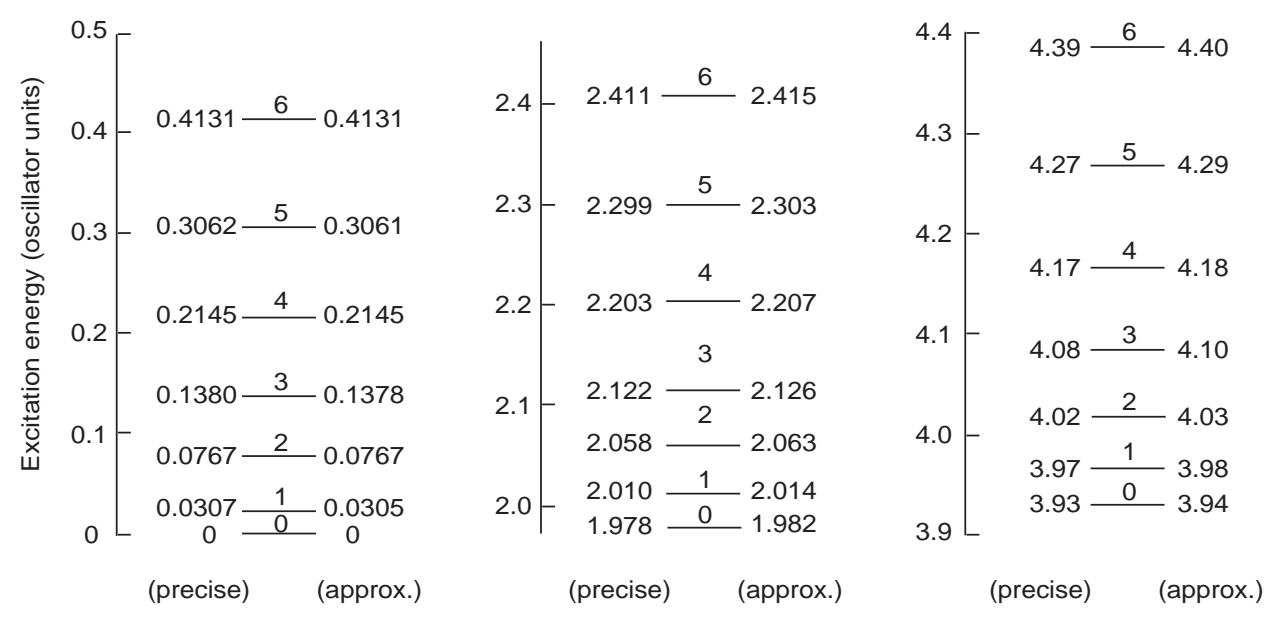

FIG. 7: Energy-level spectrum of the Hamiltonian $\hat{H}(\alpha)$ for $\alpha=1.5$ and $M=100$ for the three lowest-energy states of $\mathrm{SO}(5)$ angular momentum $v \leq 6$. The precise energies were computed by diagonalization with 100 basis states. The approximate values were computed with just 5 basis states chosen as described in the text.

\section{MORE GENERAL APPLICATIONS}

\section{A. An $N=3$ example}

In many problems of interest, the $\mathrm{SO}(N)$ rotational invariance is broken. For example, in $\mathbb{R}^{3}$, it is of interest to study splittings of molecular energy levels by a crystal field. This might be done with a Hamiltonian of the type

$$
\hat{H}=-\frac{1}{2 M} \nabla^{2}+V(r)+\chi r^{2}\left(3 \cos ^{2} \theta-1\right) .
$$

With the $M=0$ component of the $L=1$ spherical tensor $\mathcal{Q}$ given, according to equation (96), by $\mathcal{Q}_{10}=\cos \theta$, the function $3 \cos ^{2} \theta-1$ is proportional to the $M=0$ component of the $L=2$ tensor $(\mathcal{Q} \otimes \mathcal{Q})_{2}$, i.e.,

$$
3 \cos ^{2} \theta-1=\sqrt{6}(\mathcal{Q} \otimes \mathcal{Q})_{20} .
$$

The reduced matrix elements of the tensor $(\mathcal{Q} \otimes \mathcal{Q})_{2}$, obtained from those of $\mathcal{Q}$ by Racah recoupling, are given by

$$
\left\langle l_{2}\left\|(\mathcal{Q} \otimes \mathcal{Q})_{2}\right\| l_{1}\right\rangle=\sum_{l} \sqrt{5(2 l+1)} W\left(l_{1} 2, l_{2} 2 ; l 2\right)\left\langle l_{2}\|\mathcal{Q}\| l\right\rangle\left\langle l\|\mathcal{Q}\| l_{1}\right\rangle .
$$

It follows that

$$
\left\langle l_{2} m\left|\left(3 \cos ^{2} \theta-1\right)\right| l_{1} m\right\rangle=\sum_{l} \sqrt{30(2 l+1)}\left(l_{1} m, 20 \mid l_{1} m\right) W\left(l_{1} 2, l_{2} 2 ; l 2\right)\left\langle l_{2}\|\mathcal{Q}\| l\right\rangle\left\langle l\|\mathcal{Q}\| l_{1}\right\rangle,
$$

where $\left(l_{1} m, 20 \mid l_{1} m\right)$ is an $\mathrm{SO}(3)$ Clebsch-Gordan coefficient. These matrix elements are readily evaluated with the $\left\langle l^{\prime}\|\mathcal{Q}\| l\right\rangle$ matrix elements given by equation (97).

The effect on a spherical molecule of putting it into a quadrupole field is to deform it somewhat. However, if the molecule is already deformed the primary effect is to align the deformation of the molecule with the field. Thus, for a well-deformed diatomic molecule such as the $\mathrm{HCl}$ molecule, an informative way to study the spectrum and eigenfunctions of the Hamiltonian (117) would be to start by solving for the Hamiltonian

$$
\hat{H}_{0}=-\frac{1}{2 M} \nabla^{2}+V(r)+\chi r_{0}^{2}\left(3 \cos ^{2} \theta-1\right) .
$$

This will give the splittings of energy levels due to the alignment effect. Solutions for the perturbed Hamiltonian

$$
\hat{H}=\hat{H}_{0}+\chi\left(r^{2}-r_{0}^{2}\right)\left(3 \cos ^{2} \theta-1\right)
$$

will then give the added perturbations of the spectra coming from the rotation-radial vibration coupling interactions and provide information about the rigidity of the molecule. 


\section{B. An $N=5$ example}

For $N>3$, there may be terms in the Hamiltonian which break the $\mathrm{SO}(N)$ invariance but retain rotational invariance with respect to a suitably defined $\mathrm{SO}(3) \subset \mathrm{SO}(N)$ subgroup. For example, a Hamiltonian of interest in the nuclear collective model is of the form

$$
\hat{H}(\alpha)=-\frac{1}{2 M} \nabla^{2}+\frac{1}{2} M\left[(1-2 \alpha) r^{2}+\alpha r^{4}\right]+\kappa r^{3}(\mathcal{Q} \otimes \mathcal{Q} \otimes \mathcal{Q})_{0},
$$

where $(\mathcal{Q} \otimes \mathcal{Q} \otimes \mathcal{Q})_{0}$ is triple product of $\mathrm{SO}(5) \supset \mathrm{SO}(3), v=1, L=2, \mathcal{Q}$ tensors coupled to $\mathrm{SO}(3)$ angular momentum $L=0$. This $\mathrm{SO}(3)$ coupled product is the $L=0$ component of a $v=3, \mathrm{SO}(5)$ tensor.

The matrix elements of $(\mathcal{Q} \otimes \mathcal{Q} \otimes \mathcal{Q})_{0}$ are given by

$$
\begin{aligned}
\left\langle v^{\prime} \alpha^{\prime} L M\left|(\mathcal{Q} \otimes \mathcal{Q} \otimes \mathcal{Q})_{0}\right| v \alpha L M\right\rangle= & \sum_{\substack{v_{1} \alpha_{1} L_{1} \\
v_{2} \alpha_{2} L_{2}\\
}}(-1)^{L+L_{2}} \sqrt{\frac{\left(2 L_{1}+1\right)\left(2 L_{2}+1\right)}{2 L+1}} W\left(L 2 L_{2} L ; L_{1} 2\right) \\
& \times\left\langle v^{\prime} \alpha^{\prime} L\|\mathcal{Q}\| v_{2} \alpha_{2} L_{2}\right\rangle\left\langle v_{2} \alpha_{2} L_{2}\|\mathcal{Q}\| v_{1} \alpha_{1} L_{1}\right\rangle\left\langle v_{1} \alpha_{1} L_{1}\|\mathcal{Q}\| v \alpha L\right\rangle .
\end{aligned}
$$

Note, however, that the reduced matrix elements appearing in this expression are $\mathrm{SO}(3)$-reduced matrix elements; they are related to the $\mathrm{SO}(5)$-reduced matrix elements of equation (99) by the expression

$$
\left\langle v_{1} \alpha_{1} L_{1}\|\mathcal{Q}\| v \alpha L\right\rangle=\left(v \alpha L, 12 \| v_{1} \alpha_{1} L_{1}\right)\left\langle v_{1}\|\mathcal{Q}\| v\right\rangle,
$$

where $\left(v \alpha L, 12 \| v_{1} \alpha_{1} L_{1}\right)$ is an $\mathrm{SO}(5)$ Clebsch-Gordan coefficient in an $\mathrm{SO}(3)$ basis. An algorithm for computing such CG coefficients and tables of values has been given in Ref. 19 .

The low-energy level spectrum of the Hamiltonian [123), calculated with a large value of $\alpha$ [16], is shown in Fig. 8 The figure shows a ground-state rotational band and a sequence of excited (so-called gamma-) vibrational bands of the type given by the phenomenological Bohr-Mottelson nuclear collective model [15].

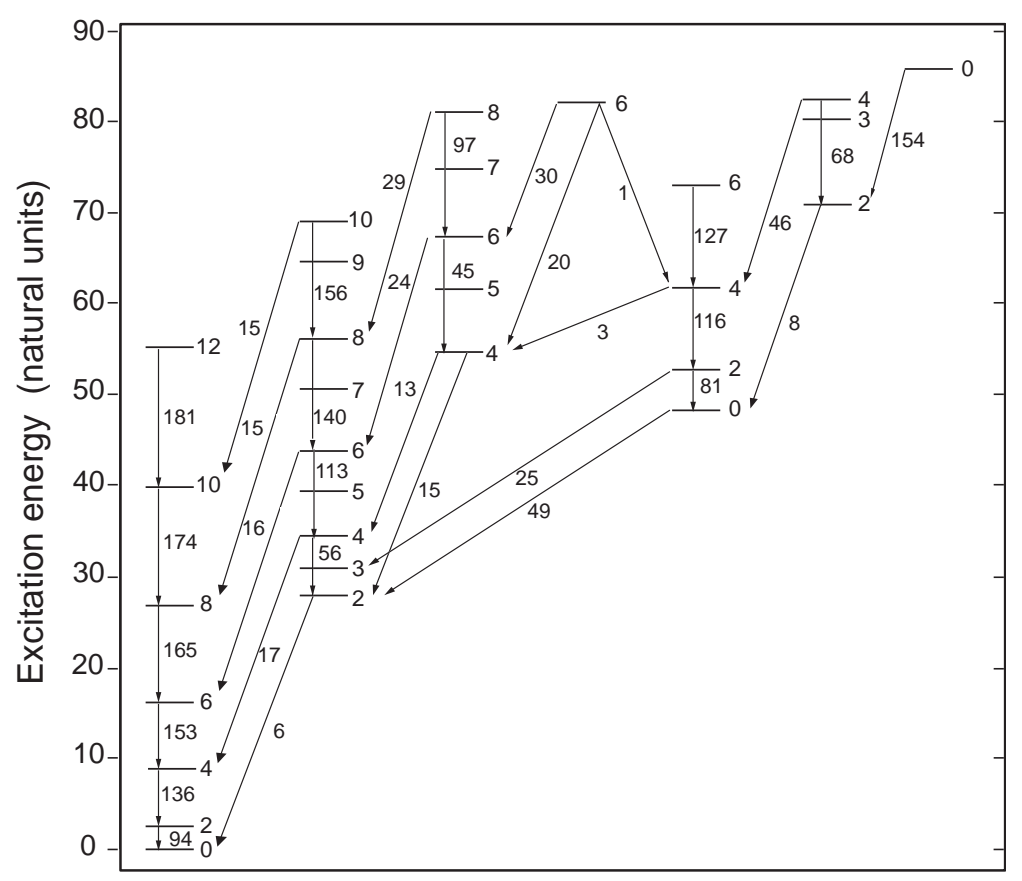

FIG. 8: Low energy-level spectrum of the Hamiltonian of equation 1123 with a large value of $\alpha$ and $\kappa=50 \sqrt{2 / 35}$. Each energy level is labelled by its $\mathrm{SO}(3)$ angular momentum; because of the interaction, the $\mathrm{SO}(5)$ angular momentum is no longer a good quantum number. Transition rates for electric quadrupole gamma-ray transitions indicated by arrows are shown in natural units beside the arrows; these transition rates are known in nuclear physics as reduced E2 transition rates. 


\section{CONCLUDING REMARKS}

It has been shown that the matrix elements of polynomial Hamiltonians on a Euclidean space $\mathbb{R}^{N}$ can be determined algebraically to within $\mathrm{SO}(N)$ Clebsch-Gordan coefficients.

The results obtained highlight the importance of algorithms for computing CG coefficients for various $\mathrm{SO}(N)$ groups. Currently these coefficients are available for $N \leq 6$. Explicit expressions for $\mathrm{SO}(3)$ coefficients were given already in the 1931 edition of Wigner's book on Group Theory [30] and can now be found in almost any book on angular momentum theory. The coefficients for $\mathrm{SO}(4)$, locally isomorphic to $\mathrm{SO}(3) \times \mathrm{SO}(3)$, are readily obtained from combinations of the $\mathrm{SO}(3)$ coefficients [1]. An algorithm for calculating the needed coefficients for $\mathrm{SO}(5)$ has recently been given [19] in an $\mathrm{SO}(3)$ basis defined by regarding the fundamental 5 -dimensional $v=1$ irrep of $\mathrm{SO}(5)$ as carrying in irreducible $L=2$ irrep of $\mathrm{SO}(3)$. This algorithm for $\mathrm{SO}(5)$ CG coefficients extends to $\mathrm{SO}(6)$ in an $\mathrm{SO}(5)$ $\supset \mathrm{SO}(3)$ basis by regarding the fundamental 6-dimensional irrep of $\mathrm{SO}(6)$ as spanning a sum of $v=0$ and $v=1$ $\mathrm{SO}(5)$ irreps and noting that the $v=0$ irrep of $\mathrm{SO}(5)$ is the trivial identity irrep. CG coefficients could undoubtedly be used with advantage for higher $\mathrm{SO}(N)$ groups. For example, models for octupole vibrations and rotations of nuclei or molecules with octupole deformations could be formulated on $\mathbb{R}^{7}$ and would require $\mathrm{SO}(7) \mathrm{CG}$ coefficients.

It will interesting to see if the techniques introduced have a parallel extension to hydrogenic and modified hydrogenic systems in light of the recent developments of Fortunato and Vitturi 31].

\section{Acknowledgments}

The author is pleased to acknowledge helpful suggestions and references from J.L. Wood and J. Karwowski.

[1] B.G. Wybourne, Classical Groups for Physicists, (Wiley, New York, 1974).

[2] J. Č́ižek and J. Paldus, Int. J. Quantum Chem. 12, 875 (1977).

[3] T.H. Cooke and J.L. Wood, Am. J. Phys. 70, 945 (2002).

[4] J.N. Huffaker and P.H. Dwivedi, "Factorization-method treatment of the perturbed Morse oscillator", J. Math. Phys. 16, 862 (1975); M.J. Inglefield, "Algebraic representations on eigenfunctions of the Rosen-Morse potential", J. Math. Phys. 28, 827 (1987); A.O. Barut, A. Inomata, and R. Wilson, "Algebraic treatment of second Pöschl-Teller, Morse-Rosen, and Eckart equations", J. Phys. A 20, 4083 (1987); E.D. Filho, "The Morse oscillator generalized from supersymmetry", J. Phys. A 21, L1025 (1988); O.L. Lange, "An operator solution for the Hultheén potential", Am. J. Phys. 59, 151 (1991).

[5] E. Schrödinger, Proc. Roy. Irish Acad. A46, 9 (1940); A46, 183 (1940); A47, 53 (1941).

[6] L. Infeld and T.E. Hull, Rev. Mod. Phys. 23, 21 (1951).

[7] K.T. Hecht, Quantum Mechanics (Springer, New York, 2000).

[8] V. Singh, S.N. Biswas, and K. Datta, Phys. Rev. D 18, 1901 (1978).

[9] M. Znojil, J. Phys. A 15, 2111 (1982).

[10] S.K. Bose and N. Gupta, Nuovo Cim. B 113, 299 (1998).

[11] J. Karwowski and L. Cyrnek, Ann. Phys. (Leipzig) 13, 181 (2004).

[12] L.E. Gendenshtein, Pis'ma Zh. Eksp. Teor. Fiz. 38, 299 (1983) [JETP Lett. 38, 356 (1983)].

[13] L. Armstrong Jr., J. Math. Phys. 12, 953 (1971).

[14] T.G. Haskell and B.G. Wybourne, Il Nuovo Cim. 12 B, 185 (1972).

[15] A. Bohr and B.R. Mottelson, Nuclear Structure, vol. II (Benjamin, Reading, 1975).

[16] D.J. Rowe, Nucl. Phys. A 735, 372 (2004).

[17] D.J. Rowe and P.S. Turner, Nucl. Phys. A 753, 94 (2005).

[18] E. Chaćon, M. Moshinsky, and R.T. Sharp, J. Math. Phys. 17, 668 (1976); E. Chaćon and M. Moshinsky, J. Math. Phys. 18, 870 (1977).

[19] D.J. Rowe, P.S Turner, and J. Repka, J. Math. Phys. 45, 2761 (2004).

[20] R. Howe, Proc. of Symp. in Pure Math. 33, 275 (1979).

[21] M. Moshinsky and C. Quesne, J. Math. Phys. 12, 1772 (1971).

[22] P.M. Davidson, Proc. Roy. Soc. 135, 459 (1932).

[23] S.G. Rohoziński, J. Srcbrny, H. Horbaczewska, Z. Physik 268, 401 (1974).

[24] J.P. Elliott, J.A. Evans and P. Park, Phys. Lett. B 169, 309 (1986).

[25] D.J. Rowe and C. Bahri, J. Phys. A: Math. Gen. 31, 4947 (1998).

[26] P.S. Turner and D.J. Rowe, Nucl. Phys. A 756, 333 (2005).

[27] S. Bell, R. Davidson, and P.A. Warsop, J. Phys. B 3, 113, 123 (1970).

[28] J.M. Arias, C.E. Alonso, A. Vitturi, J.E. García-Ramos, J. Dukelsky, A. Frank, Phys. Rev. C 68 (2003) $041302 ;$ D. Bonatsos, D. Lenis, N. Minkov, P.P. Raychev, P.A. Terziev, Phys. Rev C 69 (2004) 014302 and 044316.

[29] O.K. Vorov and V.G. Zelevinsky, Nucl. Phys. A 439, 207 (1985); V.G. Zelevinsky, J. Mod. Phys. E 2, 273 (1993). 
[30] E.P. Wigner, Gruppentheorie (Viewig, Brunswick, Germany, 1931); cf. also Group Theory and its applications to the quantum mechanics of atomic spectra (Academic press, 1959).

[31] L. Fortunato and A. Vitturi, Nucl. Part. Phys. 29, 1341 (2003). 